\title{
COVID-19 related olfactory dysfunction prevalence and natural history in ambulatory patients*
}

Daniel R. Bacon', Princess Onuorah', Alexander Murr', Christopher A. Wiesen², Jonathan Oakes³, Brian D. Thorp', Adam M. Zanation', Charles S. Ebert Jr. ', David Wohl', Brent A. Senior', Adam J. Kimple ${ }^{1,5}$

' Department of Otolaryngology/Head \& Neck Surgery, University of North Carolina Chapel Hill, Chapel Hill, NC, USA

${ }^{2}$ Odum Institute - University of North Carolina at Chapel Hill, Chapel Hill, NC, USA

${ }^{3}$ Institute for Global Health and Infectious Diseases, University of North Carolina at Chapel Hill, Chapel Hill, NC, USA

${ }^{4}$ Department of Medicine, Division of Infectious Diseases, University of North Carolina at Chapel Hill, Chapel Hill, NC, USA

${ }^{5}$ Marsico Lung Institute, Chapel Hill, NC, USA
Rhinology Online, Vol 4: 131 - 139, 2021

http://doi.org/10.4193/RHINOL/21.034

*Received for publication:

July 14, 2021

Accepted: August 3, 2021

Published: August 13, 2021

\begin{abstract}
Background: Evidence regarding prevalence of COVID-19 related Olfactory dysfunction (OD) among ambulatory patients is highly variable due to heterogeneity in study population and measurement methods. Relatively few studies have longitudinally investigated OD in ambulatory patients with objective methods.
\end{abstract}

Methods: We performed a longitudinal study to investigate OD among COVID-19 ambulatory patients compared to symptomatic controls who test negative. Out of 81 patients enrolled, 45 COVID-19 positive patients and an age- and sex-matched symptomatic control group completed the BSIT and a questionnaire about smell, taste and nasal symptoms. These were repeated at 1 month for all COVID-19 positive patients, and again at 3 months for those who exhibited persistent OD. Analysis was performed by mixed-effects linear and logistic regression.

Results: $46.7 \%$ of COVID-19 patients compared to 3.8\% of symptomatic controls exhibited OD at 1-week post diagnosis. At 1 month, 16.7\%, (6 of 36), of COVID-19 patients had persistent OD. Mean improvement in BSIT score in COVID-19 patients between 1-week BSIT and 1 month follow-up was 2.0. OD did not correlate with nasal congestion.

Conclusions: Ambulatory COVID-19 patients exhibited OD significantly more frequently than symptomatic controls. Most patients regained normal olfaction by 1 month. The BSIT is a simple validated and objective test to investigate the prevalence of OD in ambulatory patients. OD did not correlate with nasal congestion which suggests a congestion-independent mechanism of OD.

Key words: ambulatory patients, anosmia, hyposmia, olfactory disorders, olfactory dysfunction, olfactory testing, psychophysical testing, SARS-CoV-2, smell loss

\section{Introduction}

SARS-CoV-2 has become ubiquitous throughout the world, infecting more than 130 million people, and prompting the largest public health intervention in history ${ }^{(1)}$. Many containment strategies rely on early identification and isolation of infected individuals. In this regard, COVID-19 related olfactory dysfunction (OD), having been identified as an early indicator of infection by the World Health Organization (WHO) and Center for Disease Control and Prevention $(C D C)^{(2,3)}$, represents a red flag that could help clinicians, public health officials and the general public contain the spread of disease ${ }^{(4)}$.

A recent meta-analysis of nearly 30,000 patients reported a prevalence of COVID-19 related OD of $47.9 \%$ (95\% confidence interval [CI]: $41.2-54.5)^{(5)}$. Notably, further systematic review 
has revealed a wide range in reported prevalence (19-73.6\%)

${ }^{(6)}$, and furthermore, prevalence was higher among studies using objective methods $(72.1 \% \text { vs } 44.5 \%)^{(5)}$. Lima et al. and Prajapati et al. have independently found that $30-40 \%$ of patients with objectively measured OD may not actually perceive OD by self-report ${ }^{(7,8)}$. Furthermore, significant heterogeneity $\left(I^{2}:>90 \%\right)$ exists, not only between measurement methods, but also between study populations which has limited precise definition of prevalence ${ }^{(5)}$. However, Moein et al. report near universal prevalence of OD (96\%) among hospitalized patients using objective psychophysical testing ${ }^{(9)}$.

While knowledge of OD in hospitalized patients is important, understanding its manifestations in ambulatory patients bears more relevance to the pandemic response. This is especially true as these patients are in the community, have milder symptoms or are otherwise asymptomatic, and thus may not isolate themselves to prevent transmission. The reported prevalence of COVID-19 related OD among ambulatory patients has significant variation, which is in part attributable to differing measurement methods: $41.1 \%^{(10)}$ (Lee et al. survey), 66.3\% ${ }^{(11)}$ (Boscolo-Rizzo et al. survey), 68\% ${ }^{(12)}$ (Yan et al. survey), 70\% ${ }^{(13)}$ (Niklassen et al. objective threshold testing). Evidence regarding resolution of OD in ambulatory patients is mainly from survey data, though some have used threshold and psychophysical testing ${ }^{(13-16)}$. In a predominately outpatient cohort, Gorzkowski et al. used a survey to find that greater than $95 \%$ of COVID-19 patients reported improvement in olfaction by 26 days after onset, and the mean time from onset to start of recovery was 11.6 days ${ }^{(17)}$. Studies using threshold testing identified OD in $27 \%$ to $37 \%$ of patients 1-5 months after onset ${ }^{(13,14)}$.

This is a prospective longitudinal cohort study using the Brief Smell Identification Test (BSIT) in ambulatory patients with COVID-19 compared to symptomatic controls. Its findings contribute to the growing body of objectively measured olfactory literature regarding prevalence and recovery of COVID-19 related OD among ambulatory patients. Additionally, this study contributes to prognostic clarity for patients suffering from COVID-19 related OD.

\section{Materials and methods}

\section{Study design and patient population}

This study was approved by the Institutional Review Board at UNC (IRB\# 20-1992). The schedule of events is depicted in Figure 1. Patients presenting to a respiratory diagnostic center (RDC) affiliated with the University of North Carolina (UNC) Health system were prospectively enrolled in a longitudinal study. Patients were enrolled between August 3, 2020 and November 19, 2020 within 1-5 days of a SARS-CoV-2 polymerase chain reaction $(P C R)$ test. Inclusion criteria included: age $\geq 18$ years and presentation to a UNC RDC. Exclusion criteria included: hospitalization, presence of $\mathrm{OD}$ at baseline, or non-English speaking. Asymp- tomatic patients were excluded from the COVID-19 negative control group. The control group was age- and sex-matched $( \pm$ 3 years).

Participants were enrolled during a phone encounter 1-5 days after diagnosis at which point, they were specifically asked about smell loss, and were administered a past medical history questionnaire. One week after a COVID-19 test, participants were provided an enrollment packet including a 12-item Brief Smell Identification Test (BSIT), and a symptom questionnaire inquiring about nasal, olfactory and gustatory symptoms (Supplemental data). All COVID-19 positive patients were sent a follow-up BSIT and symptom questionnaire 1 month after their positive test. COVID-19 positive patients who exhibited OD received an additional BSIT and symptom questionnaire 3 months after their positive test. The age and sex matched control group all presented to a testing center with symptoms of COVID-19 but had a negative SARS-CoV-2 PCR test. They completed the BSIT and symptom questionnaire only at 1-week post diagnosis.

\section{Olfactory testing and questionnaire}

The BSIT is a 12-item psychophysical olfactory test that is validated with good test-retest reliability (Sensonics International, Haddon Heights, NJ, USA). Each correctly identified odorant from a list of four multiple choice options confers one point. The BSIT has a minimal clinically important difference (MCID) of 1 question ${ }^{(18)}$. A score $<9$ indicates $\mathrm{OD}^{(19)}$. A score $<4$ fails to surpass guesswork which may suggest complete anosmia. A score $<2$ is suggestive of malingering. Nasal, olfactory, and gustatory symptoms were assessed using a 13-question survey on a 5-point Likert scale. The olfactory and gustatory questions were derived from a validated chemosensory questionnaire for patients treated for head and neck cancer ${ }^{(20)}$. Three additional questions were included regarding nasal symptoms such as congestion, pain and drainage. The Likert scale assessed frequency of symptoms, ranging from $1=$ never, $2=$ rarely, $3=$ sometimes, 4=often, to $5=$ always.

Baseline demographics, presenting symptoms and comorbid conditions were obtained by combination of a pre-specified telephone questionnaire and medical record review. The selection of comorbid conditions was taken directly from the CDC list of high-risk conditions for COVID-19(21). The telephone questionnaire also asked about history of conditions that have potential to impair olfaction at baseline, such as head injury, sinus surgery, or allergic rhinitis.

\section{Statistical analysis}

Statistical analysis was performed by GraphPad Prism version 9 (GraphPad Software, La Jolla, CA, USA) and SAS version 9.4 (Cary, NC, USA). Descriptive statistics were used to report baseline characteristics, clinical features and comorbid conditions. These were reported as means, percentages, standard deviation (SD), 
ranges and absolute numbers.

The primary outcomes were the percentage of COVID-19 positive patients with $\mathrm{OD}$ at 1-week compared to their matched control group as well as to percentage of COVID-19 positive patients exhibiting persistent OD at 1 month follow-up. Statistical significance was set at $\mathrm{p}<0.05$.

A linear mixed-effects model was used to compare 1-week BSIT scores as continuous variables between the COVID-19 positive group and controls. Fixed effects included time and group, and time by group interaction. A random subject effect was included. The same model was also used to compare the 1-week BSIT in the COVID-19 group to BSIT at 1-month post diagnosis. A mixed effects logistic regression was used to make the same comparisons with OD as a categorical variable (BSIT $<9$ represents measurable OD).

Pearson correlation and simple linear regression was performed to assess the relationship between nasal congestion and BSIT scores in the COVID-19 positive group at 1-week. Simple linear regression was used to validate a subjective olfactory composite score derived from the symptoms questionnaire in predicting OD as measured by BSIT.

\section{Results}

81 ambulatory patients with SARS-CoV-2 positive PCR tests and 38 symptomatic but SARS-CoV-2 PCR negative controls were enrolled. Of the enrolled patients, 45 COVID-19 positive

Table 1. Baseline demographics, clinical characteristics and comorbidities.

\begin{tabular}{|c|c|c|c|c|c|}
\hline & $\begin{array}{c}\text { COVID-19 (+) Phone } \\
\text { Encounter }\end{array}$ & $\begin{array}{l}\text { COVID-19 (+) } \\
1 \text { week }^{\mathrm{a}}\end{array}$ & $\begin{array}{l}\text { COVID-19 (+) } \\
1 \text { Month }\end{array}$ & $\begin{array}{l}\text { Symptomatic } \\
\text { Controls Phone } \\
\text { encounter }\end{array}$ & $\begin{array}{l}\text { Symptomatic } \\
\text { Control } 1 \text { week }\end{array}$ \\
\hline Sample size & 81 & 45 & 36 & 38 & 26 \\
\hline Mean age, years (SD; range) & $38.21(19 ; 18-81)$ & $39.87(18 ; 18-81)$ & $39.68(18 ; 18-70)$ & $38.45(15 ; 18-71)$ & $39.38(14 ; 19-71)$ \\
\hline Sex & $52 \mathrm{~F} / 29 \mathrm{M}$ & $32 \mathrm{~F} / 13 \mathrm{M}$ & $25 \mathrm{~F} / 11 \mathrm{M}$ & $26 \mathrm{~F} / 12 \mathrm{M}$ & $20 \mathrm{~F} / 6 \mathrm{M}$ \\
\hline $\begin{array}{l}\text { Race/Ethnicity, n (\%) } \\
\text { Black } \\
\text { Caucasian } \\
\text { Hispanic } \\
\text { Unknown }\end{array}$ & $\begin{array}{c}7(8.6) \\
43(53.1) \\
8(9.9) \\
23(28.4)\end{array}$ & $\begin{array}{c}2(4.4) \\
31(68.9) \\
3(6.7) \\
9(20.0)\end{array}$ & $\begin{array}{c}2(6.1) \\
25(69.4) \\
2(5.6) \\
7(19.4 .)\end{array}$ & $\begin{array}{c}1(2.6) \\
30(78.9) \\
1(2.6) \\
6(15.7)\end{array}$ & $\begin{array}{c}0 \\
23(88.5) \\
1(3.8) \\
2(7.7)\end{array}$ \\
\hline $\begin{array}{l}\text { Self-reported olfactory } \\
\text { dysfunction during phone } \\
\text { encounter }^{b}\end{array}$ & $39(57.4)$ & $24(57.1)$ & $15(50)$ & $1(2.6)$ & $1(4)$ \\
\hline Smoking history & $10(12.7)$ & $4(8.9)$ & $5(13.9)$ & $2(4.8)$ & 0 \\
\hline Prior history of smell lossc & $7(9.5)$ & $5(11)$ & $3(8.3)$ & $5(13.5)$ & $5(20)$ \\
\hline Prior history of taste loss ${ }^{c}$ & $6(8.0)$ & $4(8.9)$ & $3(8.3)$ & $4(10.5)$ & $4(15.3)$ \\
\hline \multicolumn{6}{|l|}{ Medical/Surgical History } \\
\hline Sinusitis & $24(29.6)$ & $14(31.8)$ & $14(38.9)$ & $17(44.7)$ & $14(53.8)$ \\
\hline Allergic rhinitis & $34(42.0)$ & $20(44.4)$ & $18(50)$ & $23(60.5)$ & $19(73.1)$ \\
\hline Head trauma & $10(12.3)$ & $7(15.5)$ & $6(16.7)$ & $9(23.6)$ & $9(34.6)$ \\
\hline Nose trauma & $3(3.7)$ & $1(2.2)$ & $1(2.8)$ & $3(7.9)$ & $2(7.7)$ \\
\hline Sinus Surgery & $3(3.7)$ & $2(4.4)$ & $1(2.8)$ & $2(5.3)$ & $2(7.7)$ \\
\hline Hypertension & $12(14.8)$ & $7(15.5)$ & $7(19.4)$ & $2(5.3)$ & $1(3.8)$ \\
\hline Diabetes & $4(4.9)$ & $2(4.4)$ & 0 & 0 & 0 \\
\hline Cardiovascular disease & $4(4.9)$ & $2(4.4)$ & $3(8.3)$ & 0 & 0 \\
\hline Asthma & $8(9.9)$ & $4(8.9)$ & $4(11.1)$ & $2(5.3)$ & $2(7.7)$ \\
\hline COPD & $2(2.5)$ & $2(4.4)$ & 0 & 0 & 0 \\
\hline Pulmonary fibrosis & 0 & 0 & 0 & $1(2.6)$ & 0 \\
\hline Cystic fibrosis & 0 & 0 & 0 & 0 & 0 \\
\hline Pregnancy & $1(1.2)$ & 0 & 0 & 0 & 0 \\
\hline
\end{tabular}

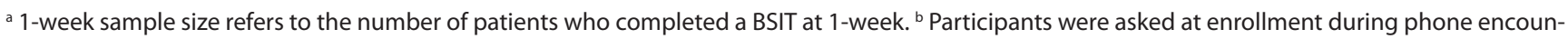
ter if they experienced smell loss at any point since the onset of symptoms. ${ }^{c}$ Prior history of smell or taste loss was determined by telephone prior to enrollment. Smell loss had to be resolved prior to onset of acute symptoms, and any patient with baseline smell loss was excluded.

Values may not add to $100 \%$ due to sporadic missingness. COVID-19= Coronavirus Disease 2019. 


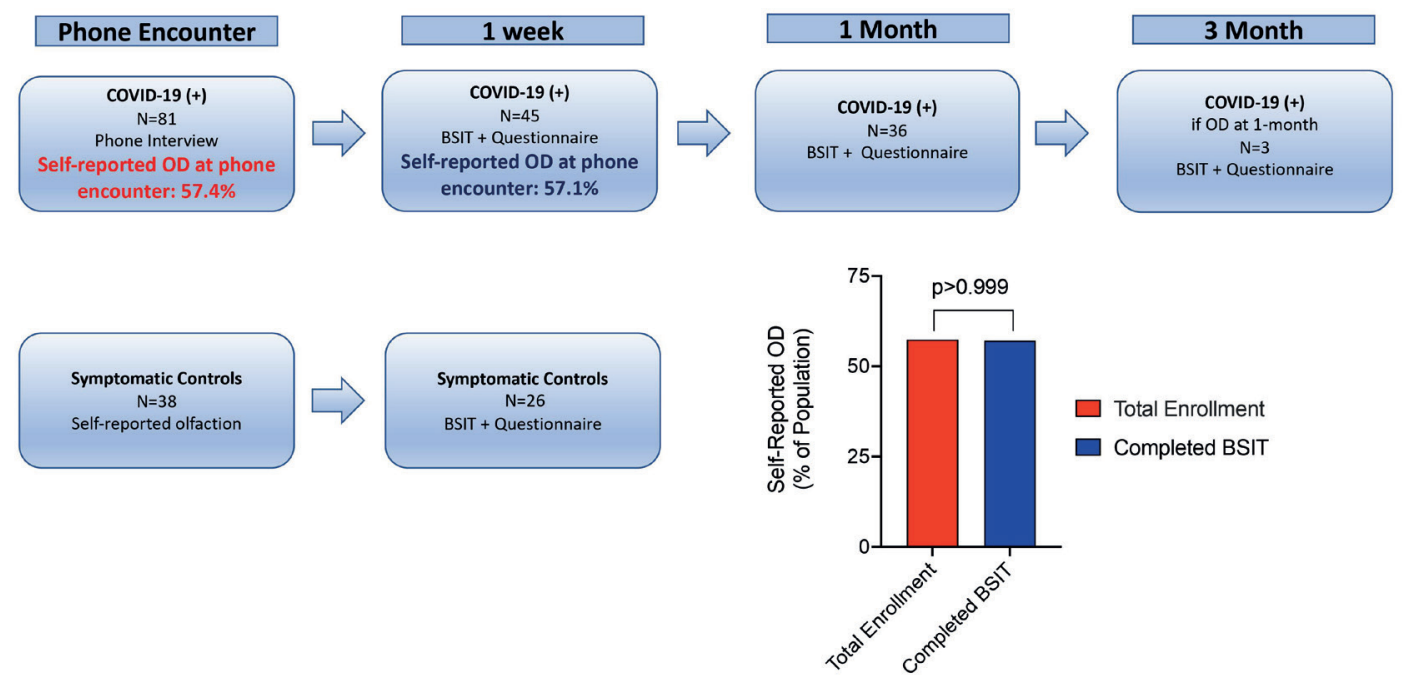

Figure 1. Schedule of Events for COVID-19 Positive and Symptomatic Control Group. Patients were enrolled by phone encounter and were asked about smell loss 1-5 days after a SARS-CoV-2 test. A cohort of COVID-19 patients and age- and sex-matched symptomatic controls were sent a BSIT and questionnaire 1-week post-diagnosis. All COVID-19 positive patients were sent a BSIT and questionnaire at 1 month, and those with persistent olfactory dysfunction (OD) at 1 month $(n=5)$, received a third BSIT and questionnaire at 3 months. The bar graph shows self-reported OD at the time of phone encounter for the total enrollment (57.4\%) in red compared to those who ultimately completed a BSIT (57.1\%) in blue suggesting patients who completed a BSIT were similar to those who did not at the time of phone encounter.
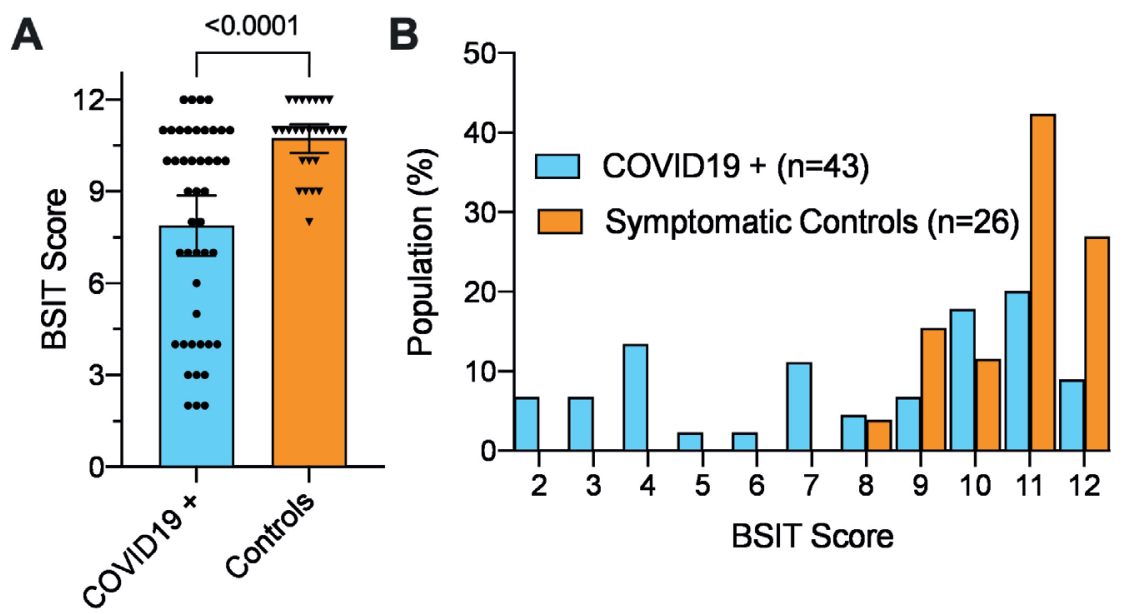

Figure 2. COVID-19 Positive Patients Have Significantly Lower BSIT Scores than Symptomatic Controls. A) The mean BSIT score at 1-week in the COVID19 group (blue) was 7.9 ( $95 \% \mathrm{Cl} 7.1$ - 8.6) compared to 10.7 (95\% Cl $9.7-11.7)$ in the control group (orange), resulting in a difference of -2.9 ( $95 \% \mathrm{Cl}$ -4.1 to $-1.6, p<0.001)$. Each participant score is represented by a dot. Error bars represent $95 \%$ confidence intervals. B) The same BSIT scores are plotted as a percentage of the population. Among abnormal BSIT scores in the COVID-19 group ( $n=21), 28.6 \%(n=6)$ were in the lowest quartile (score of $0-3)$, with an additional $38.0 \%(n=8)$ in the second to lowest quartile (score of 4-6). An additional $33.3 \%(n=7)$ exhibited mild OD (score of 7-8).

patients completed the 1-week BSIT and questionnaire and 36 participants completed a 1-month BSIT. 5 participants who had persistent smell loss at 1 month were sent a 3 month follow up BSIT, and 3 of these 5 participants completed the test. 26 symptomatic controls who presented to a RDC but were SARSCoV-2 PCR test negative, completed a 1-week BSIT and symptom questionnaire. These results as well as baseline demographics, comorbidities and clinical characteristics are shown in Table 1.
Figure 1 depicts the schedule of events. Despite significant attrition, patients completing a BSIT reported very similar rates of smell loss at the time of phone encounter compared to the total enrollment (57.4 vs 57.1\%; p>0.999, Figure 1).

Comparison of 1-week BSIT Scores in COVID-19 positive patients vs symptomatic controls

In the COVID-19 positive cohort, the mean time between 


\section{A}

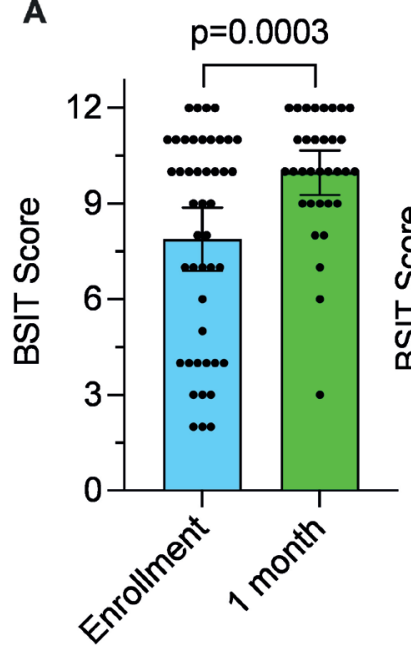

B

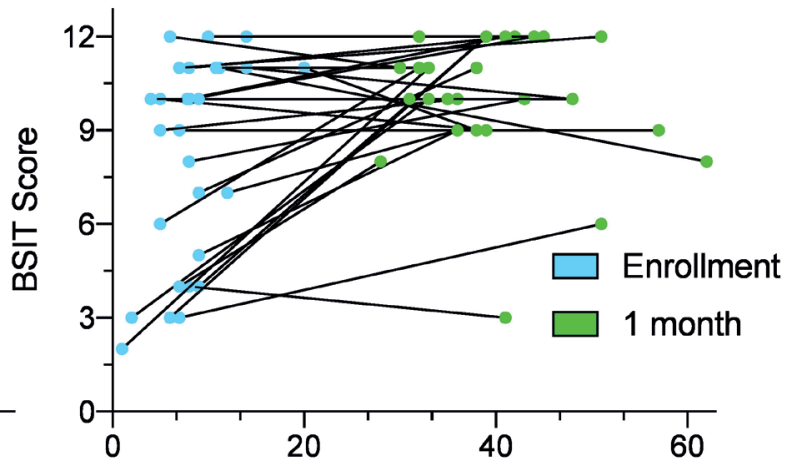

Days from Positive COVID-19 PCR Test

Figure 3. BSIT Score improved significantly in COVID-19 patients between 1 week and 1 month. A) BSIT Score improved by 2.0 ( $95 \% \mathrm{Cl} 1.0-3.0)$ from 7.9 (95\% Cl 7.1 - 8.6) to 9.9 (95\% Cl 9.0 - 10.7) in COVID-19 patients at baseline (blue) compared to 1 month later (green). B) Shows improvement in BSIT score (y-axis) from baseline (blue) to one month (green) with days from COVID-19 test on the $x$-axis. A score $<9$ indicates OD. The average time between COVID-19 test and 1-week BSIT was 8.9 days (95\% CI 7.4 - 10.4). The average time from COVID-19 test to follow-up BSIT was 38.4 days $(95 \%$ $\mathrm{Cl} 35.6-41.1)$.
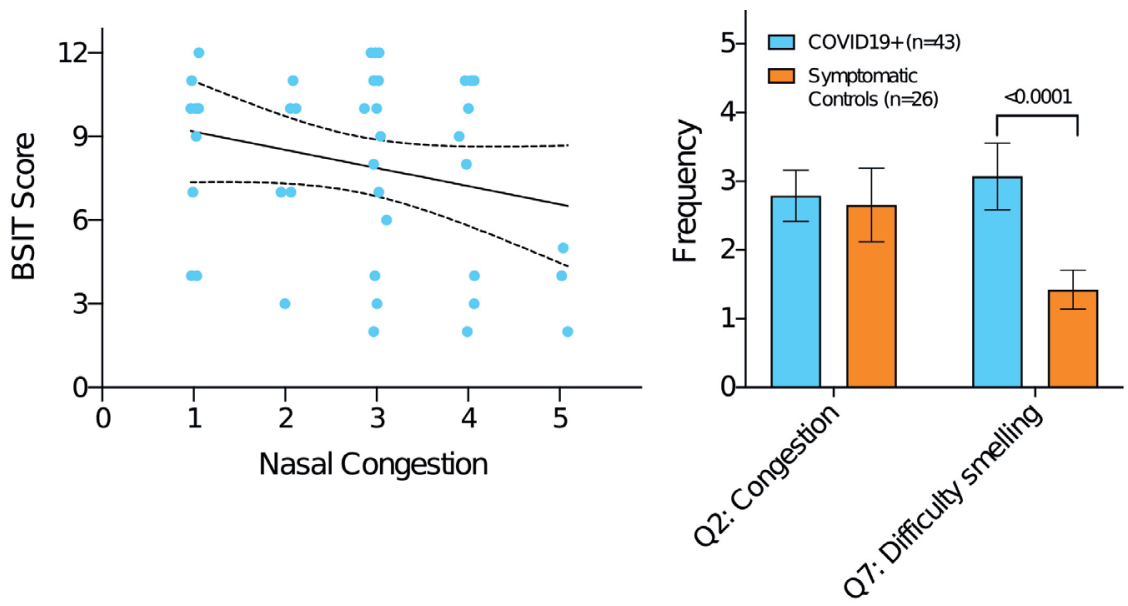

Figure 4. BSIT Scores do not correlate with congestion COVID-19 patients suggesting a congestion independent mechanism of OD. A) There was no significant correlation between BSIT scores in COVID-19 patients and self-reported nasal congestion $(r=-0.233, p=0.13)$. The simple linear regression (solid line) is plotted with $95 \%$ confidence interval bands (dotted lines), (slope $=-0.638,95 \% \mathrm{Cl}-1.48$ to 0.203 ). Simulated noise was performed to display overlapping points. B) There was no significant difference between self-reported congestion in COVID-19 patients vs symptomatic controls $(0.137, p=0.66)$. COVID-19 patients had self-reported increased difficulty smelling $(-1.65, p<0.001)$. Error bars represent $95 \%$ confidence intervals. The questionnaire used a 5-point Likert scale (1=never, 2=rarely, 3=sometimes, 4=often, 5=always). Q2: I have had nasal congestion. Q7: It has been hard for me to smell.

COVID-19 test and 1 week time point for BSIT completion was 8.9 days $(95 \% \mathrm{Cl} 7.4-10.4)$ compared to 8.2 days (95\% Cl 7.1-9.2) in the control cohort. At 1 week, $46.7 \%$ (21 of 45 ) of COVID-19 participants had measurable OD (BSIT<9) compared to $3.8 \%(1$ of 26) of controls $(p<0.001)$. The mean BSIT score at 1-week in the COVID-19 group was 7.9 out of a total of 12 (95\% CI $7.1-8.6)$ compared to $10.7(95 \% \mathrm{Cl} 9.7-11.7)$ in the control group, resulting in a difference of $-2.9(95 \% \mathrm{Cl}-4.1$ to $-1.6, \mathrm{p}<0.001$; Figure 2 , panel A). As above, previous studies have validated a minimal clinically important difference of $1.0^{(18)}$. There were no significant differences in sinonasal symptom burden, including frequency of nasal pain, drainage, or congestion, between COVID-19 patients and symptomatic controls (Figure S1).

Stratified BSIT scores among COVID-19 patients and symptomatic controls are shown in Figure 2, panel B. Among COVID-19 patients who did exhibit OD ( $n=21), 28.6 \%$, $(n=6)$ demonstrated 
scores in the lowest quartile, (0-3). An additional 38.0\% $(n=8)$ had slightly better scores (4-6), and $33.3 \%(n=7)$ of patients demonstrated mild OD (7-8). The only patient in the control cohort who exhibited OD had a BSIT score of 8.

\section{Longitudinal olfactory outcome}

In the COVID-19 positive cohort, the mean time between the COVID-19 test date and follow-up BSIT completion was 38.4 days (95\% Cl 35.6-41.1: Figure 3, Panel B).At follow-up, 16.7\% (6 of 36) in the COVID-19 cohort had persistent OD. The follow-up BSIT in COVID-19 patients improved by a clinically significant 2.0 (95\% Cl 1.0 - 3.0, p<0.001; Figure 3, Panel A), from $7.9(95 \% \mathrm{Cl}$ $7.1-8.6)$ to $9.9(95 \% \mathrm{Cl} 9.0-10.7)$.

\section{Questionnaire and self-reported nasal congestion}

Results of the questionnaire, which can be found in the supplementary file, demonstrated significant differences in smell and taste related symptoms, and notably there were no significant differences in nasal symptoms between COVID-19 group and symptomatic controls (Figures S1-S6). COVID-19 positive patients exhibited no correlation between the 1-week BSIT and the frequency of patient reported nasal congestion $(r=-0.233$, $p=0.13$; slope $=-0.638,95 \% \mathrm{Cl}-1.48$ to 0.203 ; Figure 4, Panel A). Furthermore, there was no significant difference between the frequency of self-reported congestion in COVID-19 patients compared to controls, while there was a significant difference in self-reported difficulty with smell $(1.65, \mathrm{p}<0.0001$; Figure 4, Panel B).

\section{Discussion}

This study prospectively compared olfactory function between ambulatory patients with COVID-19 and symptomatic controls who tested negative. Additionally, we longitudinally followed the COVID-19 positive cohort for 3 months to assess recovery of olfaction.

This study used objective psychophysical testing to investigate the incidence of COVID-19 related OD in ambulatory patients compared to symptomatic patients who test negative $(46.7 \%$ vs. $3.8 \%, \mathrm{p}<0.001)$. Stratified BSIT scores revealed that about onethird of patients with olfactory dysfunction had complete anosmia (score 0-3), and about two-thirds were confined to the lower 2 quartiles of olfactory scores (scores 0-6; Figure 2, Panel B). Our longitudinal findings indicate that a great majority of $\mathrm{CO}$ VID-19 ambulatory patients will recover normal olfaction after 1 month $(83.3 \%)$, and this may be underestimated due to attrition. Recovery of olfaction may represent the clinical consequence of the basic pathophysiologic mechanism of COVID-19-related OD, which is thought to be secondary to SARS-CoV-2 infection of the supporting sustentacular cells, which express ACE2 and TMPRSS2, and not the olfactory neurons directly(22). This mechanism may also explain why we did not find any correlation between BSIT scores and the frequency of nasal congestion; whereas many other pathogens cause transient OD by mechanical obstruction of the olfactory cleft through nasal congestion. Our results clinically support the basic hypothesis that SARSCoV-2 causes OD independent of nasal congestion (Figure 4). This study has several limitations. Firstly, it would be ideal to obtain olfactory testing earlier and more frequently following COVID-19 diagnosis. This potentially could have identified patients who had OD but recovered prior to their first BSIT; this also would have more precisely delineated the natural history of OD.

Additionally, there is risk for selection bias for study entry and selection bias by attrition, as patients with smell loss may be more interested in participating than patients without chemosensory complaints. However, this appears to be minimal given similar baseline characteristics between patients who enrolled, and those who were lost to follow-up; most importantly, the rates of self-reported OD at the time of phone encounter in COVID-19 positive patients who completed a BSIT compared to the entire enrollment were very similar, (57.4\% vs $57.1 \%$; $p>0.999$, Figure 1).

\section{Conclusions}

The precise rate of OD among ambulatory patients with COVID-19 is unknown. Incidence of OD is significantly higher than in patients with similar symptoms who test negative for COVID-19. Although our study was not designed to correlate temporality between the onset of OD and infectivity, it is reasonable from these findings to advise ambulatory patients with new onset OD to quarantine and be tested for COVID-19. Additionally, this study provides further prognostic clarity for clinicians to be used in counseling patients that the great majority of ambulatory patients who experience COVID-19 related OD recover during the first month after diagnosis.

\section{List of abbreviations}

BSIT = Brief Smell Identification Test; $C D C=$ Center for Disease Control and Prevention; $\mathrm{Cl}=$ Confidence interval; COVID-19= Coronavirus Disease 2019; IRB = Institutional Review Board; $M C I D=$ Minimal Clinically Important Difference; OD = Olfactory dysfunction; $\mathrm{PCR}=$ Polymerase chain reaction; $\mathrm{RDC}=$ Respiratory Diagnostic Center; SARS-CoV-2 = Severe Acute Respiratory Syndrome Coronavirus 2; SD = Standard deviation; UNC = University of North Carolina; WHO = World Health Organization

\section{Authorship contribution}

AK, BS and DB designed the study protocol. DB, PO, AM, and JO consented participants, performed data collection, or arranged logistics.DB and CS performed statistical analysis. DB drafted the initial manuscript. Critical revisions were provided by all authors and the final version of the manuscript was approved by all 
authors.

\section{Acknowledgments}

Not applicable.

\section{Funding}

The project described was supported by NIH grants KL2TR002490 to AJK and the Howard Holderness Distinguished Medical Scholars program to DRB. The content is solely the responsibility of the authors and does not necessarily represent the official views of the $\mathrm{NIH}$.

\section{Ethics approval and consent to participate}

This study was approved by the Institutional Review Board at UNC (IRB\# 20-1992).

\section{Consent for publication}

Not applicable.

\section{Availability of data and materials}

The datasets used and/or analyzed during the current study are available from the corresponding author on reasonable request.

\section{Conflict of interest}

The authors report no competing interests, financial or otherwise.

\section{References}

1. WHO Coronavirus Disease (COVID-19) Dashboard. WHO.int. https://covid 19 who.int/. Last modified February 1, 2021. Accessed on April 15, 2021.

2. Coronavirus. WHO.int. https://www.who. int/health-topics/coronavirus\#tab=tab_3. Accessed on April 15, 2021.

3. Symptoms of Coronavirus. CDC.gov. https:// www.cdc.gov/coronavirus/2019-ncov/ symptoms-testing/symptoms.html. Last modified December 22, 2020. Accessed April 15, 2021

4. Ottaviano G, Carecchio M, Scarpa B, Marchese-Ragona R. Olfactory and rhinological evaluations in SARS-CoV-2 patients complaining of olfactory loss. Rhinology. 2020;58(4):400-1

5. Saniasiaya J, Islam MA, Abdullah B Prevalence of Olfactory Dysfunction in Coronavirus Disease 2019 (COVID19): A Meta-analysis of 27,492 Patients. Laryngoscope. 2020; 131(4): 865-878.

6. Santos REA, Da Silva MG, Monte Silva MCB et al. Onset and duration of symptoms of loss of smell/taste in patients with COVID19: A systematic review. Am J Otolaryngol. 2021;42(2):102889.

7. Lima MA, Silva MTT, Oliveira RV, et al. Smell Dysfunction in COVID-19 Patients: More than a Yes-No Question. J Neurol Sci. 2020; 418: 117107.

8. Prajapati DP, Shahrvini B, MacDonald $B V$, et al. Association of Subjective Olfactory Dysfunction and 12-item Odor Identification Testing in Ambulatory COVID19 Patients. Intl. Forum Allergy Rhinol. 2020; 10: 1209-1217.

9. Moein ST, Hashemian SMR, Tabarsi P, Doty RL. Prevalence and Reversibility of Smell Dysfunction Measured Psychotropically in a Cohort of COVID-19 Patients. Intl. Forum
Allergy Rhinology. 2020; 10(10): 1127-1135.

10. Lee DJ, Lockwood J, Das P, et al. SelfReported Anosmia and Dysgeusia as Key Symptoms of Coronavirus Disease 2019. CJEM. 2020; 22(5): 595-602.

11. Boscolo-Rizzo P, Borsetto D, Fabbris C, et al. Evolution of Altered Sense of Smell or Taste in Patients with Mildly Symptomatic COVID-19. JAMA Otolaryngol Head Neck Surg. 2020; 146(8): 729-732.

12. Yan $\mathrm{CH}$, Faraji F, Prajapati, et al. Association of Chemosensory Dysfunction and COVID19 in Patients Presenting with Influenza-Like Symptoms. Intl. Forum Allergy Rhinol. 2020; 10(7): 806-813.

13. Niklassen AS, Draf J, Huart C, et al. COVID19: Recovery from Chemosensory Dysfunction. A Multicentre study on Smell and Taste. Laryngoscope. 2021; 131(5): 1095-1100.

14. Le Bon SD, Pisarski N, Verbeke J, et al Psychophysical evaluation of chemosensory functions 5 weeks after olfactory loss due to COVID-19: a prospective cohort study on 72 patients. Eur Arch Otorhinolaryngol. 2021; 278(1): 101-108.

15. Bertlich M, Stihl C, Lusebrink E et al. The course of subjective and objective chemosensory dysfunction in hospitalized patients with COVID-19: a 6-month followup. Eur Arch Otorhinolaryngol. 2021: 10;1-7.

16. Speth MM, Singer-Cornelius T, Oberle $M$, Gengler I, Brockmeier SJ, Sedaghat AR. Time scale for resolution of olfactory dysfunction in COVID-19. Rhinology. 2020;58(4):404-5.

17. Gorzkowski V, Bevilacqua S, Charmillion A et al. Evolution of Olfactory Disorders in COVID-19 Patients. Laryngoscope. 2020; 130(11): 2667-2673

18. Levy JM, Mace JC, Bodner TE, et al. Defining the minimal clinically important difference for olfactory outcomes in the surgical treatment of chronic rhinosinusitis. Intl. For Allergy Rhinol. 2017; 7(8): 821-826.

19. Doty $R L$, Marcus $A$, Lee $W W$, et al. Development of the 12-item CrossCultural Smell Identification Test (CC-SIT). Laryngoscope. 1996; 106:353-6.

20. Goldberg AN, Shea JA, Deems DA, et al. A ChemoSensory Questionnaire for Patients Treated for Cancer of the Head and Neck. Laryngoscope. 2005; 115(12): 2077-86.

21. People with Certain Medical Conditions. CDC.gov. https://www.cdc.gov/ coronavirus/2019-ncov/need-extra-precautions/people-with-medical-conditions.html. Last updated December 21, 2020. Accessed July 15, 2020.

22. Brann DH, Tsukahara T, Weinreb C, et al. Non-Neuronal Expression of SARS-CoV-2 Entry Genes in the Olfactory System Suggests Mechanisms Underlying COVID19-Associated Anosmia. Sci Adv. 2020; 6(31): 5801.

Adam J. Kimple, MD, PhD

Department of Otolaryngology/Head

\& Neck Surgery

170 Manning Drive, CB 7070

Houpt Building

Chapel Hill

NC 27599-7070

Tel: +1-(984) 974-6484

Fax: +1-(919) 966-7941

E-mail: Adam_Kimple@med.unc.edu

ISSN: 2589-5613 / @2021 The Author(s). This work is licensed under a Creative Commons Attribution 4.0 International License. The images or other third party material in this article are included in the article's Creative Commons license, unless indicated otherwise in the credit line; if the material is not included under the Creative Commons license, users will need to obtain permission from the license holder to reproduce the material. To view a copy of this license, visit http://creativecommons.org/licenses/by/4.0/ 


\section{SUPPLEMENTARY DATA}

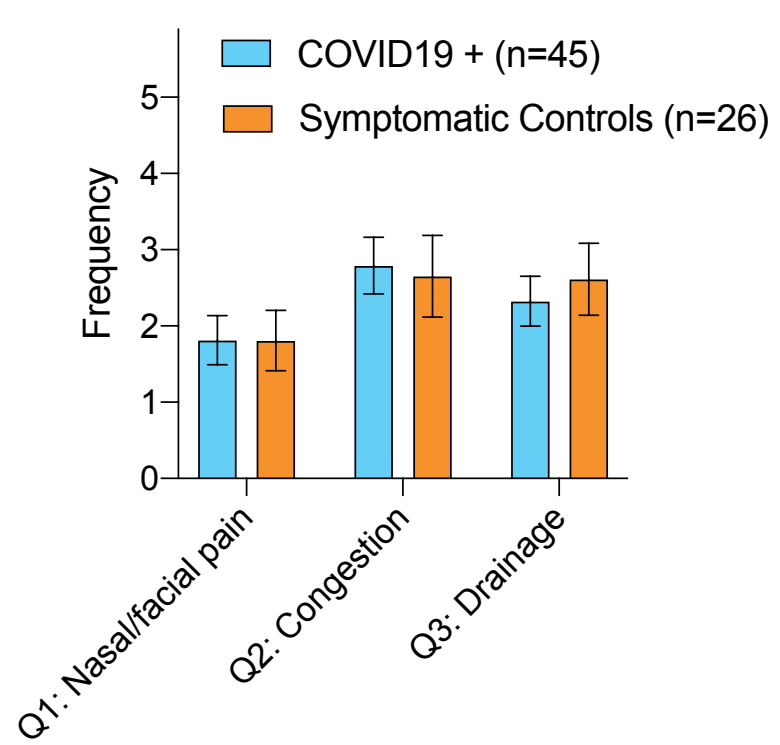

Figure S1. Nasal Symptoms were Similar Between COVID-19 + Patients and Symptomatic Controls at Baseline Indicating Comparable Illness Severity Between Groups. There was no significant difference in selfreported nasal symptoms between COVID-19+ patients (blue) and symptomatic controls (orange). Error bars represent $95 \%$ confidence intervals. Questions are answered on a 5-point Likert scale, ( $1=$ never, 2=rarely, 3=sometimes, 4=often, 5=always). Q1: Nasal/facial pain has bothered me. Q2: I have had nasal congestion. Q3: I have had nasal drainage.

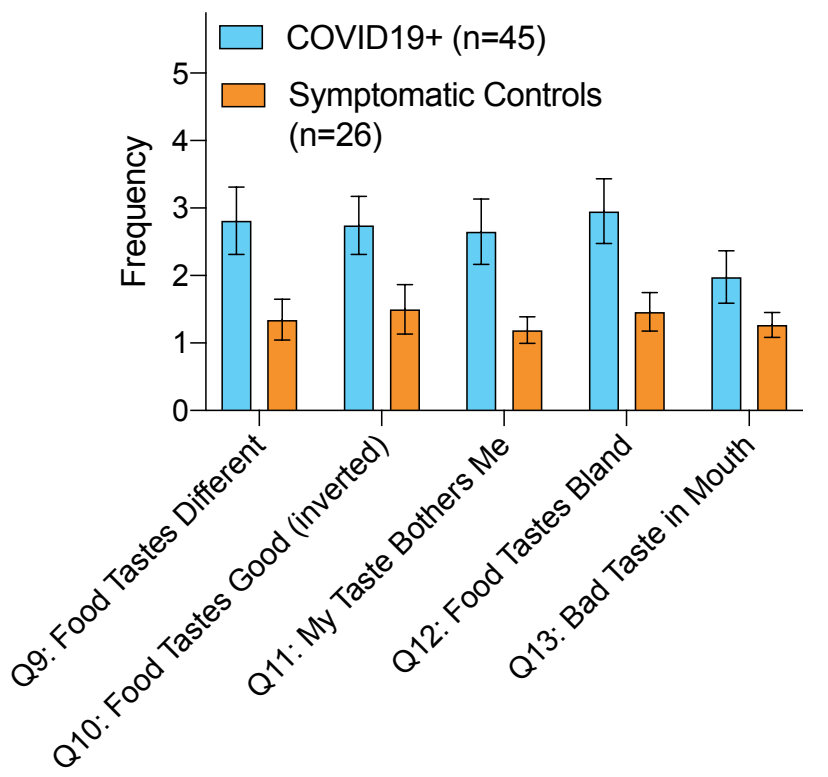

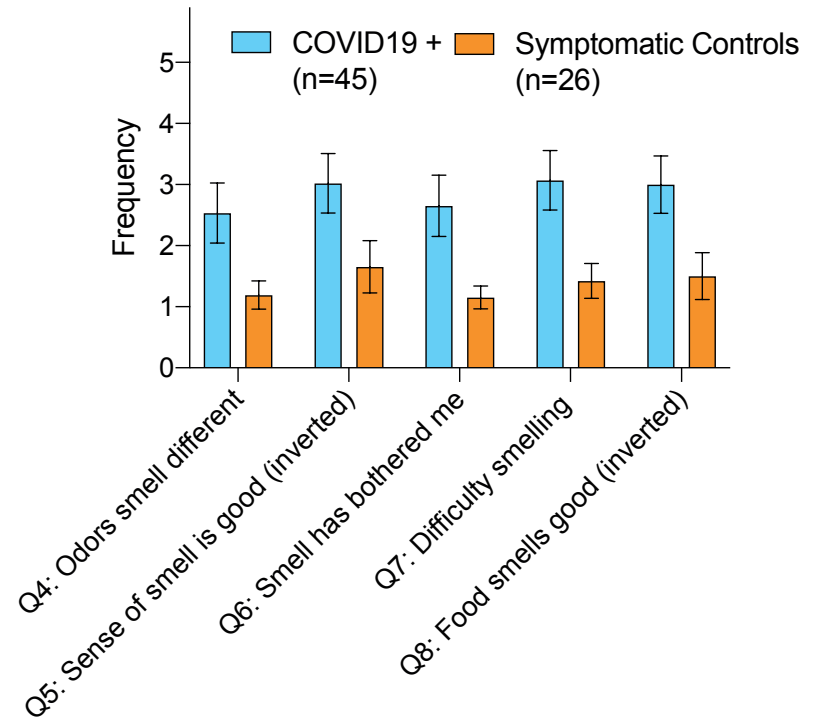

Figure S2. COVID-19 + Patients Reported Higher Frequency of Olfactory Disturbance Symptomatic Controls at Baseline. There was a significant difference in self-reported smell symptoms between COVID-19+ patients (blue) and symptomatic controls (orange). Error bars represent $95 \%$ confidence intervals. Questions are answered on a 5-point Likert scale, ( $1=$ never, $2=$ rarely, $3=$ =sometimes, $4=$ often, 5=always). Q4: Odors smell different than I remember. Q5: My smell has been good. Q6: My sense of smell has bothered me. Q7: It has been hard for me to smell. Q8: Food has smelled good. The scores for Questions 5 and 8 were inverted for consistency.

Figure S3. COVID-19 + Patients Reported Higher Frequency of Gustatory Disturbance than Symptomatic Controls at Baseline. There was a significant difference in self-reported taste symptoms in COVID-19 patients (blue) compared to symptomatic controls (orange). Error bars represent 95\% confidence intervals. Questions are answered on a 5-point Likert scale ( $1=$ never, $2=$ rarely, $3=$ sometimes, $4=$ often, $5=$ always). Q9: Food tastes different than I remember. Q10: Food has tasted good. Q11: My sense of taste has bothered me. Q12: Food has tasted bland. I have a bad taste in my mouth. Question 10 was inverted for consistency. 


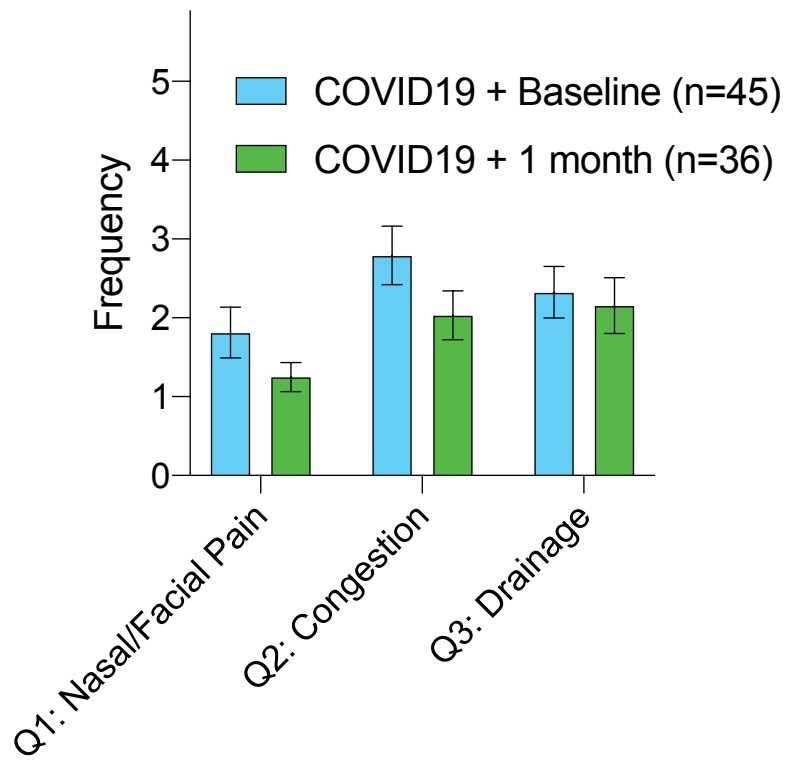

Figure S4. COVID-19 + Patients Reported Improved Nasal/Facial Pain and Congestion 1 month Compared to Baseline. There was a significant improvement in self-reported nasal/facial pain and congestion in COVID-19 + patients at baseline (blue) compared to 1 month later (green). There was no significant difference in nasal drainage. Error bars represent $95 \%$ confidence intervals. Questions are answered on a 5 -point Likert scale ( $1=$ never, $2=$ rarely, $3=$ sometimes, $4=$ often, 5=always). Q1: Nasal/facial pain has bothered me. Q2: I have had nasal congestion. Q3: I have had nasal drainage.

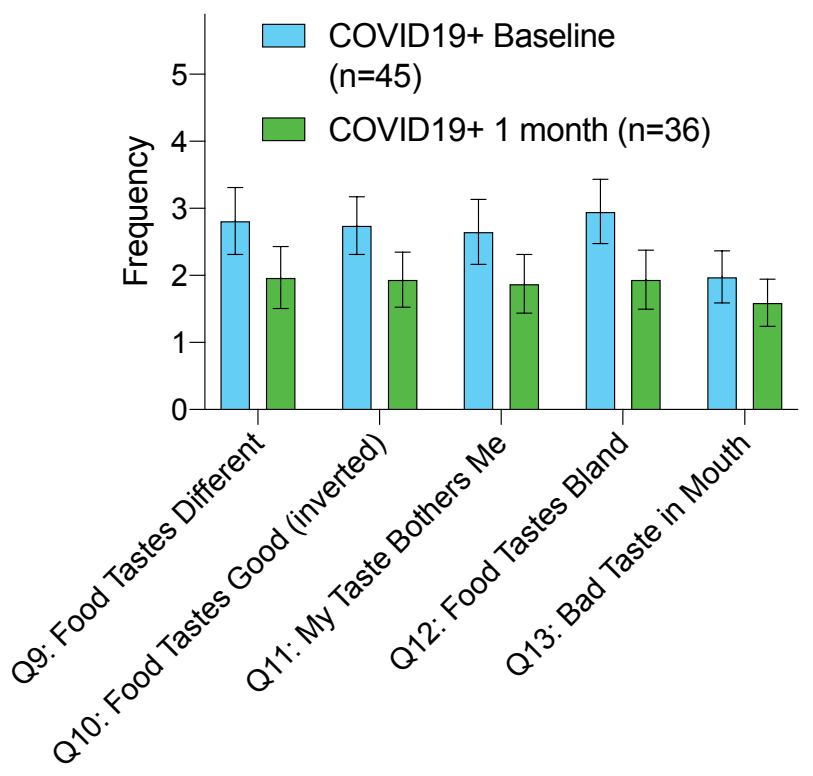

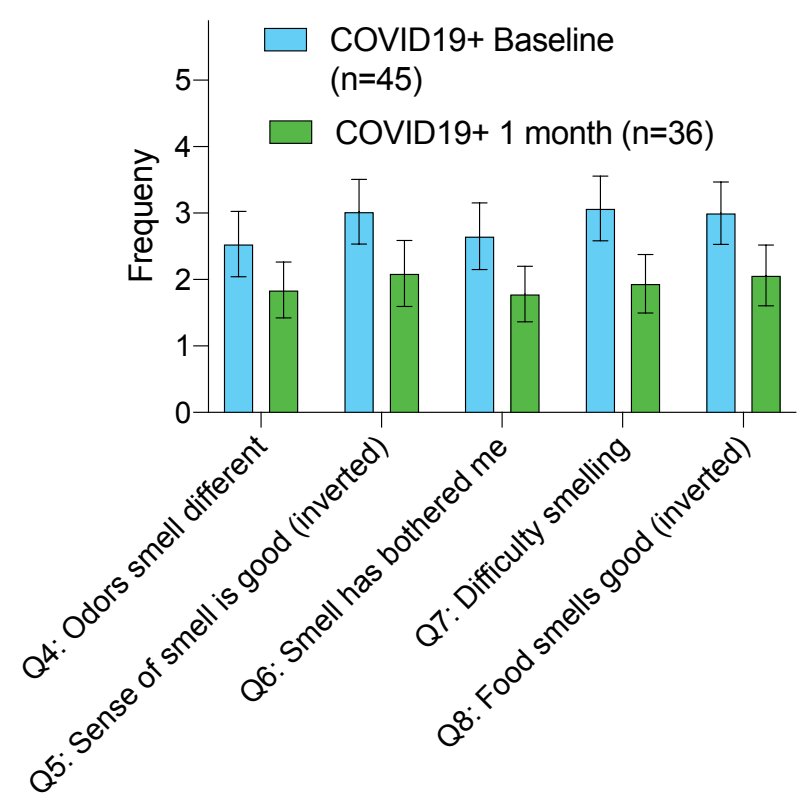

Figure S5. COVID-19 + Patients Reported Improved Olfactory Function at 1 month Compared to Baseline. There was a significant difference in self-reported smell symptoms in COVID-19 + patients at baseline (blue) compared to 1 month later (green). Error bars represent 95\% confidence intervals. Questions are answered on a 5-point Likert scale ( $1=$ never, 2=rarely, 3=sometimes, 4=often, 5=always). Q4: Odors smell different than I remember. Q5: My smell has been good. Q6: My sense of smell has bothered me. Q7: It has been hard for me to smell. Q8: Food has smelled good. The scores for Questions 5 and 8 were inverted for consistency.

Figure S6. COVID-19 + Patients Reported Improved Gustatory Function at 1 Month Compared to Baseline. There was a significant difference in self-reported taste symptoms between COVID-19 + patients at baseline (blue) compared to 1 month later (green) in all categories except Q13: I have a bad taste in my mouth. Error bars represent $95 \%$ confidence intervals. Questions are answered on a 5 -point Likert scale ( $1=$ never, 2=rarely, 3=sometimes, 4=often, 5=always). Q9: Food tastes different than I remember. Q10: Food has tasted good. Q11. My sense of taste has bothered me. Q12: Food has tasted bland. I have a bad taste in my mouth. Question 10 was inverted for consistency. 